\title{
Prevalence and Socioeconomic inequalities trends in child health comparing within and between group inequalities: Food insecurity and malnutrition in Zimbabwe
}

\section{CURRENT STATUS: POSTED}

Research Square

\section{Akim Tafadzwa Lukwa}

University of Cape Town School of Public Health and Family Medicine

च tafadzwalukwa@gmail.comCorresponding Author

ORCiD: https://orcid.org/0000-0003-2534-3731

Aggrey Siya

College of veterinary medicine and biosecurity, makerere university

Karen Nelwin Zablon

National Institute for Medical Research Muhimbili Research Centre

James Azam

DST-NRF Center of Excellence in Epidemiology and Analysis

Olufunke A. Alaba

University of Cape Town School of Public Health and Family Medicine

DOI:

10.21203/rs.3.rs-17905/v1

\section{SUBJECT AREAS}

Nutrition \& Dietetics Health Policy

\section{KEYWORDS}

Food Insecurity, Child Health, Malnutrition, Socioeconomic Inequalities 
Abstract

Background

Globally nations are advocating for universal health coverage which argues for health access for all however, inequalities in child health remain a threat to this global initiative. Even though malnutrition and food insecurity are now dominating the global development agenda, there are substantial gaps on literature about patterns and trends of socioeconomic inequalities in food insecurity and malnutrition in many developing countries. Globally an estimated 3.1 million children die annually as a result of undernutrition, shockingly sub-Saharan Africa accounts for majority of the most nutritionally insecure and food insecure children in the world. In previous decades' prevalence of stunting in Zimbabwe has been erratic. This paper assessed socioeconomic inequalities in child health focusing on malnutrition and food insecurity in Zimbabwe.

\section{Methods}

The study used Demographic Health Survey (DHS) data sets of 2010111 and 2015. Food insecurity in children was determined based on the WHO dietary diversity score. The study adopted the WHO dietary diversity score informed by the Infant and Young Child Feeding (IYCF) practices. Minimum dietary diversity as an indicator for food security is defined by a cut- off point of $>4$, therefore for this study children with less than 3 of the 13 food groups were defined as food insecure. Malnutrition was assessed using weight-for-age Z-scores, with children whose weight-for-age Z-score below minus two standard deviations (-2 SD) from the median considered malnourished. Concentration indices were computed to understand if malnutrition was dominant among the poor or rich. The paper used the Theil index, which is a generalized entropy measure and decomposed the indices by population subgroups (geographical clusters and socioeconomic status) so as to separate total inequality in the distribution between the selected groups and remaining within-group inequalities.

\section{Results}

For the period under review malnutrition prevalence increased by 1.03 percentage points (p.p) [2010/11(3.73\%); 2015(4.76\%)], while food insecurity prevalence decreased by 4.35 p.p [2010/11(78.29\%);2015(73.94)]. Prevalence of malnutrition and food insecurity increased by; 9.6p.p 
and 2p.p among poor children \& 10.23p.p and 0.5p.p among rural children. Concentration indices showed that; children from wealthy households were more likely to be food secure (pro-rich) while, children from poor households were more likely to be nutritious (pro-poor). For nutrition status socioeconomic inequality gaps appear to be widening as the concentration indices between the two time periods increased, while for food security status socioeconomic inequality gaps appear to be contracting as the concentration indices between the two time periods reduced. Decomposed Theil indices by geographical clusters (urban \& rural) for; food security status shows contracting socioeconomic inequality gaps in both geographical clusters (urban \& rural), while for nutrition status the Theil indices reflect widening socioeconomic inequality gaps among urban children and contracting socioeconomic inequality gaps among rural children.

\section{Conclusion}

The study concluded within-group inequalities to be driving most of the socioeconomic inequalities in nutritional status and food security status of children in Zimbabwe. Therefore, Zimbabwean government should design policies that focus on addressing within-group inequalities and direct food security interventions for food insecure children through availing food aid.

\section{Full Text}

Due to technical limitations, full-text HTML conversion of this manuscript could not be completed. However, the manuscript can be downloaded and accessed as a PDF.

\section{Figures}




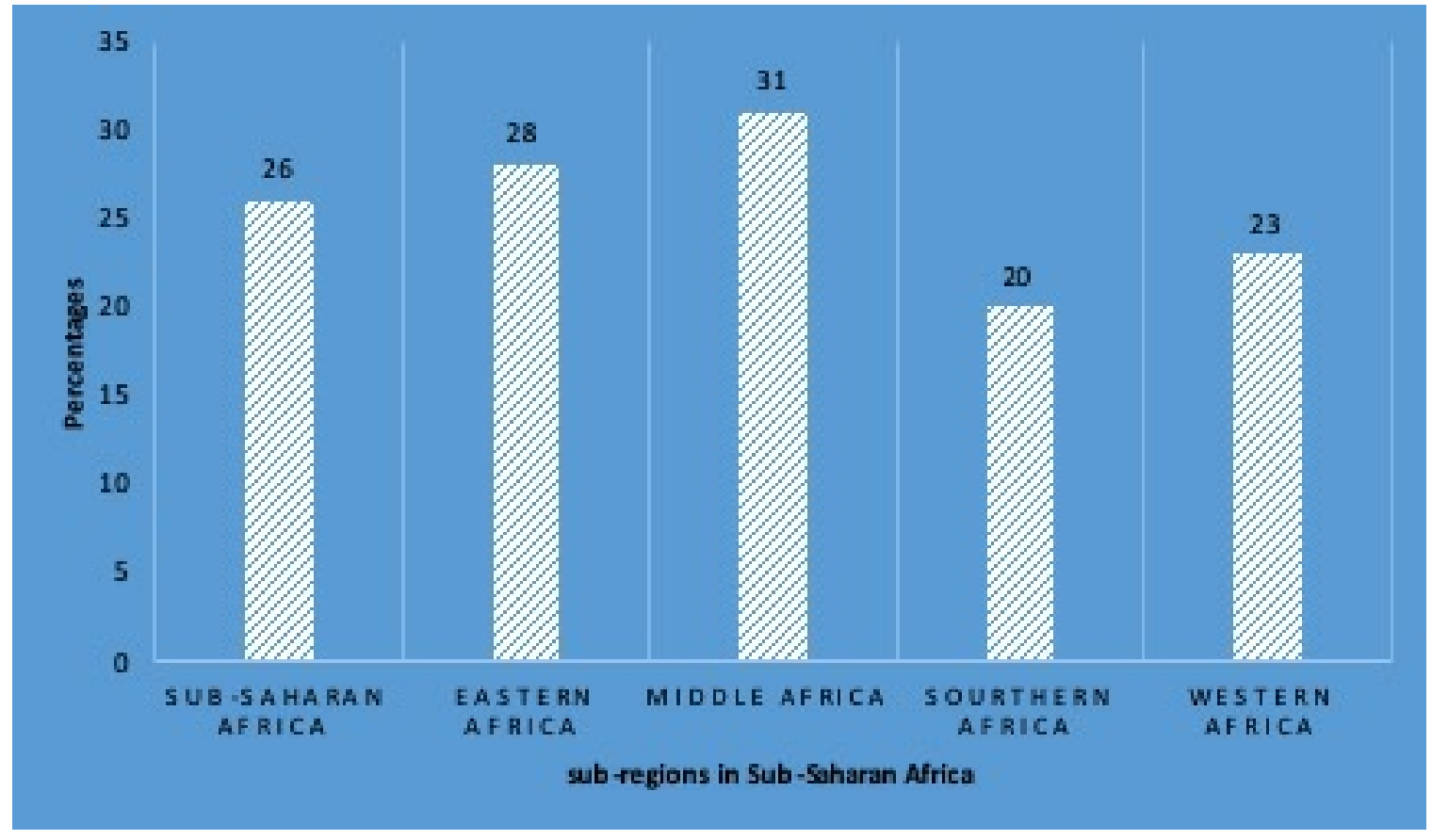

Figure 1

Prevalence of child food insecurity across sub-regions in Sub-Saharan Africa

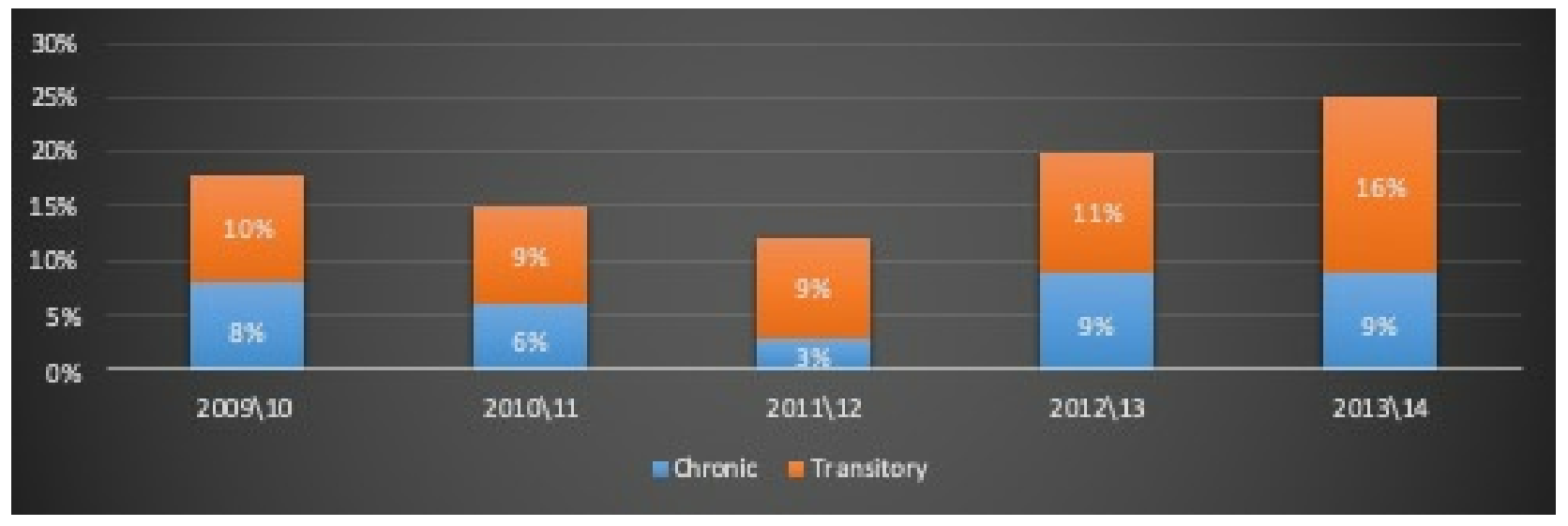

Figure 2

Estimate of chronically and transitorily food insecure rural population in Zimbabwe 2009-

2014 


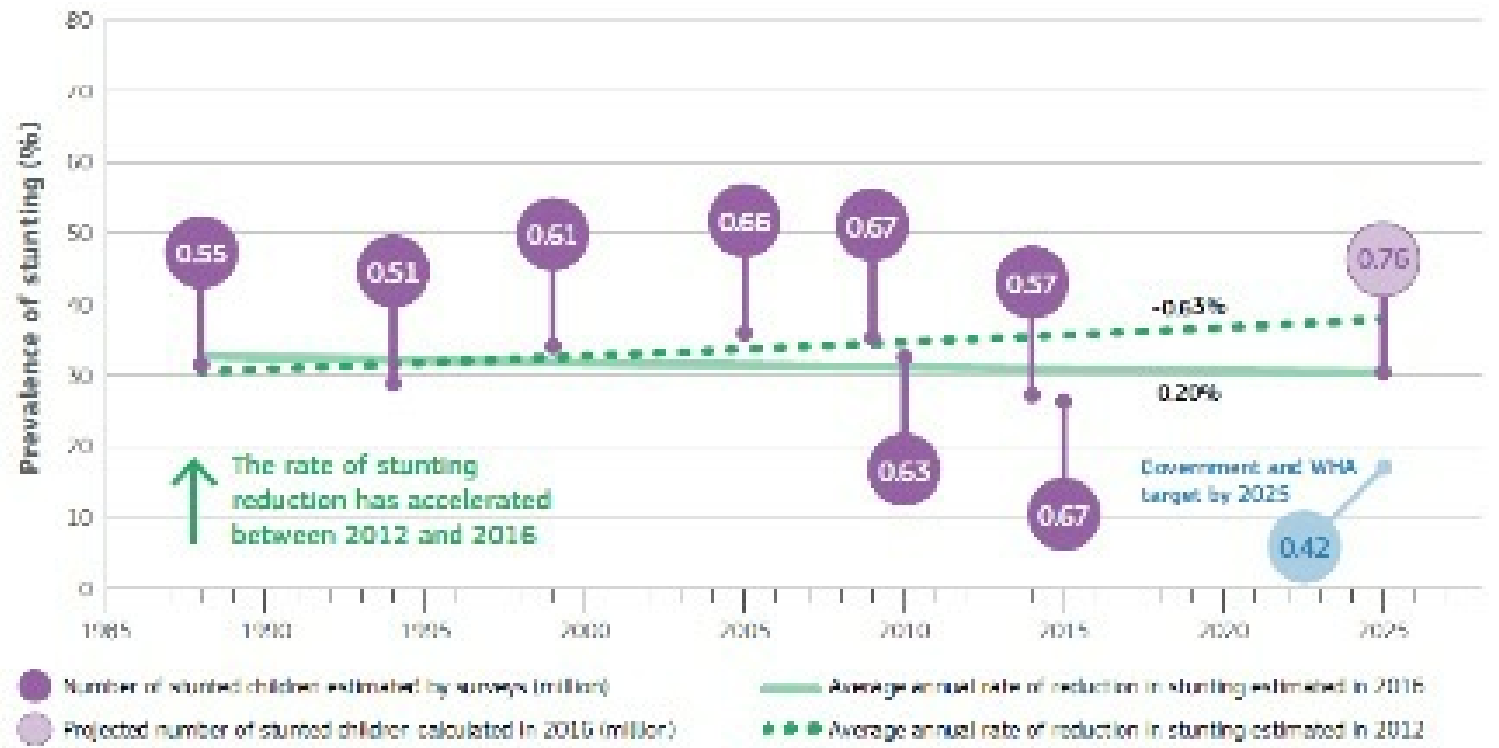

Figure 3

Trend, projection and targets in the prevalence and number of children (under-five) stunted for Zimbabwe
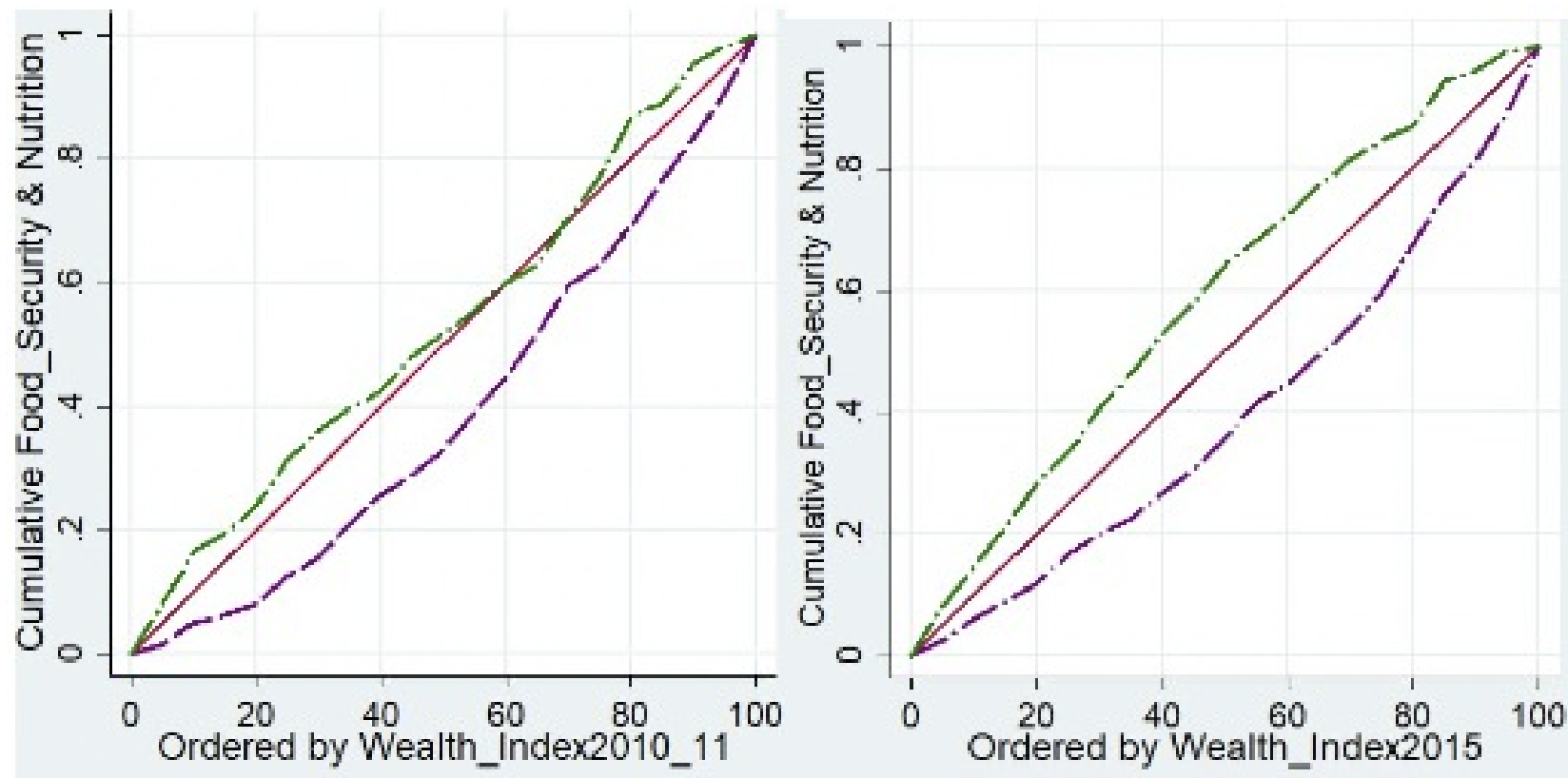

- - - Food_security_status2010_11 Nutrition_status2010_11

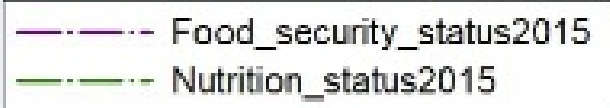

Figure 4

4a: 3b; Concentration curves for food security and malnutrition for 2010/11 and 2015 